\title{
Barriers and Facilitators to the Use of Medications for Opioid Use Disorder: a Rapid Review
}

\author{
Katherine Mackey, MD, MPP, Stephanie Veazie, MPH, Johanna Anderson, MPH, \\ Donald Bourne, MPH, and Kim Peterson, MS
}

Evidence Synthesis Program (ESP) Coordinating Center, VA Portland Health Care System, Portland, OR, USA.

\begin{abstract}
BACKGROUND: Despite evidence that medications to treat opioid use disorder (OUD) are effective, most people who could benefit from this treatment do not receive it. This rapid review synthesizes evidence on current barriers and facilitators to buprenorphine/naloxone and naltrexone at the patient, provider, and system levels to inform future interventions aimed at expanding treatment. METHODS: We systematically searched numerous bibliographic databases through May 2020 and selected studies published since 2014. Study selection, data abstraction, coding of barriers and facilitators, and quality assessment were first completed by one reviewer and checked by a second.
\end{abstract}

RESULTS: We included 40 studies of buprenorphine (5 also discussed naltrexone). Four types of patient and provider-level barriers to OUD medication use emerged-stigma related to OUD medications, treatment experiences and beliefs (positive or negative), logistical issues (time and costs as well as insurance and regulatory requirements), and knowledge (high or low) of OUD and the role of medications. Stigma was the most common barrier among patients, while logistical issues were the most common barriers among providers. Facilitators for both patients and providers included peer supports. Most administrator-identified or system-level barriers and facilitators fit into the category of logistical issues. We have moderate confidence in buprenorphine findings but low confidence in naltrexone findings due to the small number of studies.

DISCUSSION: Stigma, treatment experiences, logistical issues, and knowledge gaps are the main barriers associated with low utilization of OUD medications. These barriers can overlap and mutually reinforce each other, but given that, it is plausible that reducing one barrier may lead to reductions in others. The highest priority for future research is to evaluate interventions to reduce stigma. Other priorities for future research include better identification of barriers and facilitators for specific

Prior Presentations The work is based on a report that is available on the ESP website at https://www.hsrd.research.va.gov/publications/esp/ reports.cfm. The report was presented during an HSR\&D Cyberseminar on August 27, 2019.

Electronic supplementary material The online version of this article (https://doi.org/10.1007/s11606-020-06257-4) contains supplementary material, which is available to authorized users.

Received February 7, 2020

Accepted September 18, 2020

Published online November 3, 2020 populations, such as those with OUD related to prescription opioids, and for naltrexone use.

PROTOCOL REGISTRATION：PROSPERO; CRD42019133394

KEY WORDS: opioid use disorder; buprenorphine; naltrexone; barriers; facilitators.

J Gen Intern Med 35(Suppl 3):S954-S63

DOI: $10.1007 /$ s11606-020-06257-4

(C) Society of General Internal Medicine (This is a U.S. government work and not under copyright protection in the U.S.; foreign copyright protection may apply) 2020

\section{BACKGROUND}

Although medications (buprenorphine/naloxone, extendedrelease naltrexone, and methadone) to treat opioid use disorder (OUD) are effective, most people who could benefit from this treatment do not receive it. ${ }^{1,2}$ OUD medications remain underutilized despite federal initiatives ${ }^{3}$ and legislation designed to increase access to OUD treatment, including the 2014 enactment of major provisions of the Affordable Care Act (ACA) and the 2016 Comprehensive Addiction and Recovery Act (CARA). Multiple interconnected factors at the patient, provider, and system levels likely contribute to the continuing underuse of OUD medications. A 2019 National Academies of Science, Engineering, and Medicine consensus report based on expert opinion and informed by a literature search (not limited to the current era) cited stigma, inadequate training, and system-level factors such as insurance coverage and reimbursement as key barriers. ${ }^{2}$ To the best of our knowledge, our review is the first to systematically examine the most recent evidence on barriers and facilitators to OUD medication use in the context of increased public awareness of the opioid crisis and federal policy changes already in place.

This manuscript summarizes findings of a rapid systematic review ${ }^{4}$ and updated literature search on barriers and facilitators to OUD medication use at the patient, provider, and system levels conducted by the Department of Veterans Affairs Evidence Synthesis Program (VA ESP). This review was requested by VA Health Services Research \& Development (HSR\&D) to inform a 2019 conference on opioid safety. We focused on evidence related to buprenorphine/naloxone (referred to as buprenorphine for brevity) and naltrexone, as these 
medications can be prescribed by non-specialized providers in any setting including primary care and therefore have the greatest potential for increased use.

\section{METHODS}

Our review was guided by current standard systematic review methods and guidelines. ${ }^{5-7}$ To accommodate a rapid timeline, we streamlined certain systematic review steps, including using sequential instead of independent dual review processes. The complete description of our methods can be found on the PROSPERO international prospective register of systematic reviews (http://www.crd.york.ac.uk/PROSPERO/; registration number CRD42019133394) and in our full report. ${ }^{4}$ All steps were first completed by one reviewer and checked by a second to minimize bias and error. Disagreements were resolved by consensus or assesement by a third reviewer.

\section{Search Strategy and Study Selection}

We searched MEDLINE, PsycINFO, CENTRAL, Cochrane Database of Systematic Reviews, and EMBASE using terms for OUD, buprenorphine, naltrexone, barriers, and facilitators from database inception through May 2020, limiting the search to published and indexed articles of human subjects in English. See Appendix A for our complete search strategies. To prioritize evidence reflecting recent federal policy changes (i.e., enactment of major provisions of the ACA including parity for mental health treatment) and heightened public awareness of OUD, we subsequently limited the selection of studies to those published since 2014 . We included quantitative and qualitative studies of non-pregnant adults in the US aimed at identifying barriers and facilitators to initial use of buprenorphine and/or naltrexone or factors associated with the use of these medications. We excluded studies of methadone only and studies of OUD treatment retention (rather than initiation). See Appendix B for detailed descriptions of our inclusion and exclusion criteria.

\section{Data Abstraction and Quality Assessment}

We abstracted data from all studies, including study characteristics, setting, population, type of medication discussed, and barriers/facilitators identified (Appendix C). We conducted content analysis of reported barriers and facilitators using coding based on an iterative process without pre-defined categories. As we reviewed studies, we grouped content into existing categories or created a new category. We later identified subcategories within each of the major categories. All coding was first conducted by one reviewer and checked by a second. We classified barriers/facilitators according to whose perspective was represented in the study (e.g., barriers/facilitators reported by patients are "patient-identified barriers").

We assessed study quality using criteria adapted from Cochrane Collaboration and the Johanna Briggs Institute ${ }^{8,9}$ to evaluate whether studies met minimum methodological quality standards. For each study, we assessed (1) sampling methods, (2) adequacy of survey or interview questions in capturing the desired information, (3) reporting of population descriptions, (4) reporting of setting descriptions, and (5) methods of identifying barriers and facilitators. For quantitative studies, we also assessed (6) whether appropriate statistical analyses were conducted, and for qualitative studies, we assessed (7) whether the study used a formal process for recording, transcribing, and coding themes from interviews or open-ended responses. We categorized studies as either "met minimum criteria" if a study met all criteria \#1-5 and either \#6 or \#7 or "did not meet minimum criteria" if not (Appendix D). Data from abstraction and quality assessment were managed in Excel.

\section{Strength of Evidence and Data Synthesis}

We informally considered the strength of the evidence using principles adapted from the AHRQ Methods Guide for Comparative Effectiveness Reviews 5 , which incorporates 4 key domains: risk of bias, consistency, directness, and precision. While AHRQ's strength of evidence tool is designed to evaluate intervention studies, we felt the principles could be applied to this evidence base of non-intervention studies. For example, we evaluated risk of bias using the quality assessment method described above and consistency based on whether studies identified the same or similar barriers or facilitators. We synthesized evidence qualitatively and did not perform quantitative synthesis due to limited data or heterogeneity (i.e., variability in the perspective represented, barrier/facilitator definition, and measurement approach taken).

\section{RESULTS}

Among 3840 potentially relevant citations, 40 studies met our inclusion criteria (Fig. 1). ${ }^{1}{ }^{10-48}$ Twenty-four studies were quantitative (e.g., surveys or analyses of databases), 14 were quantitative (e.g., interviews), and two were mixed-methods (e.g., nominal group technique or surveys and interviews). We identified 11 studies $^{1,17,21-23,31,37-39,41,46}$ of patient-level barriers and facilitators and 14 studies $^{11-15,18,19,24,26-30,45}$ of provider-level barriers and facilitators. In addition, we identified 2 studies of addiction counselors (who treat patients with OUD but are not responsible for prescribing medication), ${ }^{10,32}$ 1 study of community corrections employees, ${ }^{47}$ and 1 study of a mix of healthcare professionals, substance use treatment providers, law enforcement agents, and judicial officials. ${ }^{43}$ Eleven studies evaluated system-level barriers and facilitators to OUD medication use. ${ }^{16,20,25,33-36,40,42,44,48}$ All studies examined barriers and facilitators to use of buprenorphine; five also discussed naltrexone. ${ }^{11,25,39,41,43}$ Half of studies (22 studies, 55\%) met our minimum quality criteria (Appendix C). 


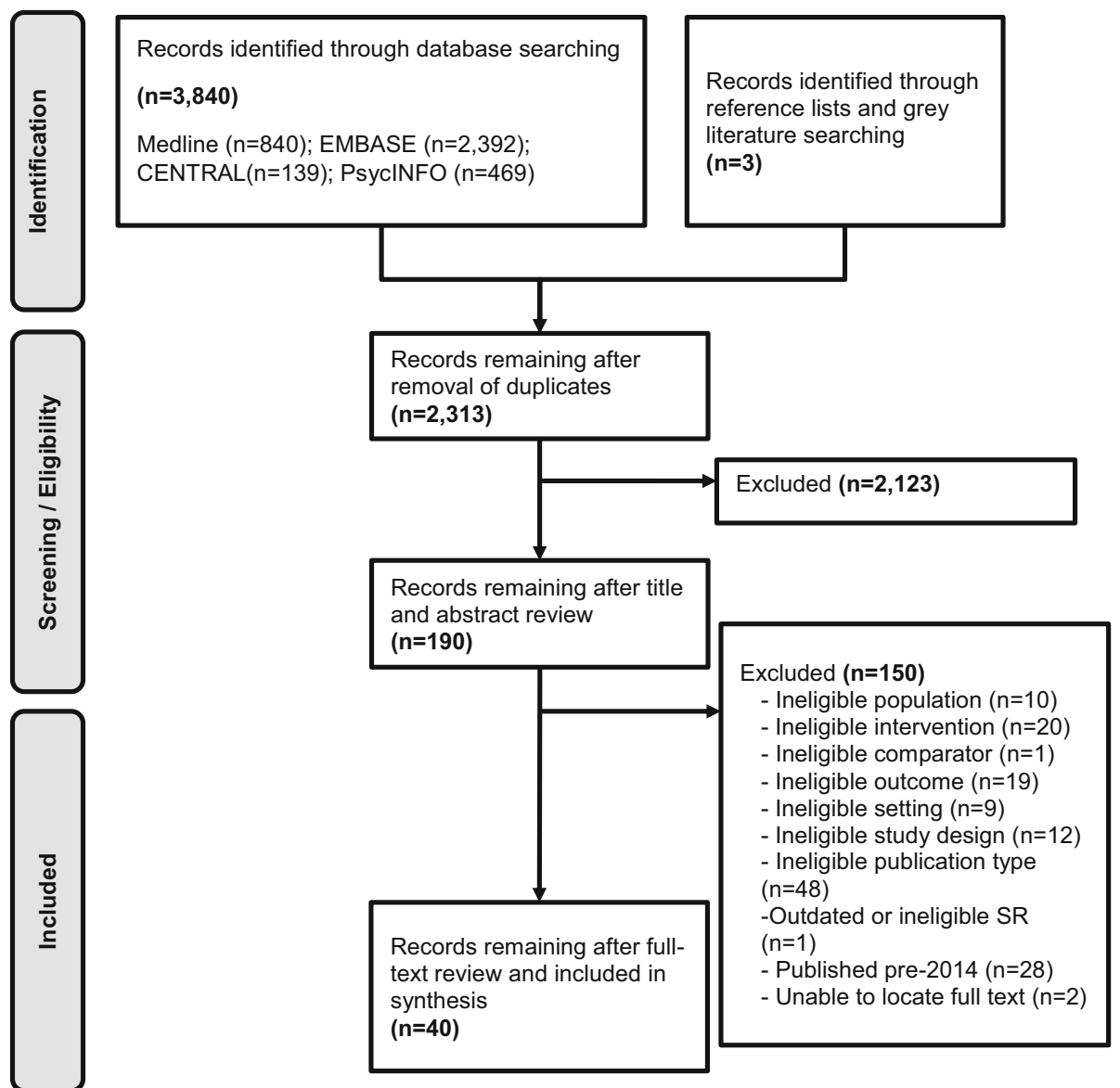

Figure 1 Literature flowchart.

Barriers to OUD medication identified by patients and providers can be grouped into 4 categories - stigma related to using or prescribing OUD medications, treatment experiences and beliefs (positive or negative), logistical issues (which we considered to include practical issues such as time and costs as well as insurance and regulatory requirements), and knowledge (high or low) of OUD and the role of medications in OUD treatment. Most administrator-identified or system-level barriers and facilitators fit into the category of practical or logistical issues. Figure 2 graphically represents how frequently different types of barriers were identified among studies from the patient, provider, and administrator or system perspective. In this manuscript, we describe or display barriers and facilitators identified by patients, providers, or systems that have the potential to be addressed by an intervention (i.e., we do not describe characteristics such as patient race or provider credentials that are associated with treatment initiation as they are unlikely to be targeted by an intervention).

\section{Strength of Evidence}

We have moderate confidence in findings on buprenorphine and low confidence in the findings on naltrexone. Overall, while only half of studies met our minimum quality criteria, results are direct (i.e., representative of the perspectives of interest) and largely consistent across studies. Our confidence is lower for naltrexone as we only identified 5 studies. While additional research is needed to understand specific barriers/ facilitators for naltrexone, more studies of buprenorphine are unlikely to reveal barriers/facilitators that are unrelated to those in this review.

\section{Patient-Identified Barriers to OUD Medication Use}

Table 1 provides an overview of patient-identified barriers and facilitators. Patient studies identified stigma as a barrier to starting OUD medications, although the impact of stigma on OUD treatment decisions varied among studies and patients and was a less frequently reported concern among treatmentengaged patients in New Haven, CT. ${ }^{39}$ Stigma can manifest in different ways including social stigma (not wanting to be associated with OUD treatment) and self or internalized stigma (shame related to having an addiction and needing treatment). ${ }^{23}$ Stigma regarding OUD medication, specifically buprenorphine, can also manifest as the view that using medication is "a crutch" and that people on buprenorphine are not really sober. ${ }^{21-23}$

Patients also identified prior experience with OUD treatment as a barrier to starting OUD medications, particularly if experiences were negative. Two qualitative studies of patients 


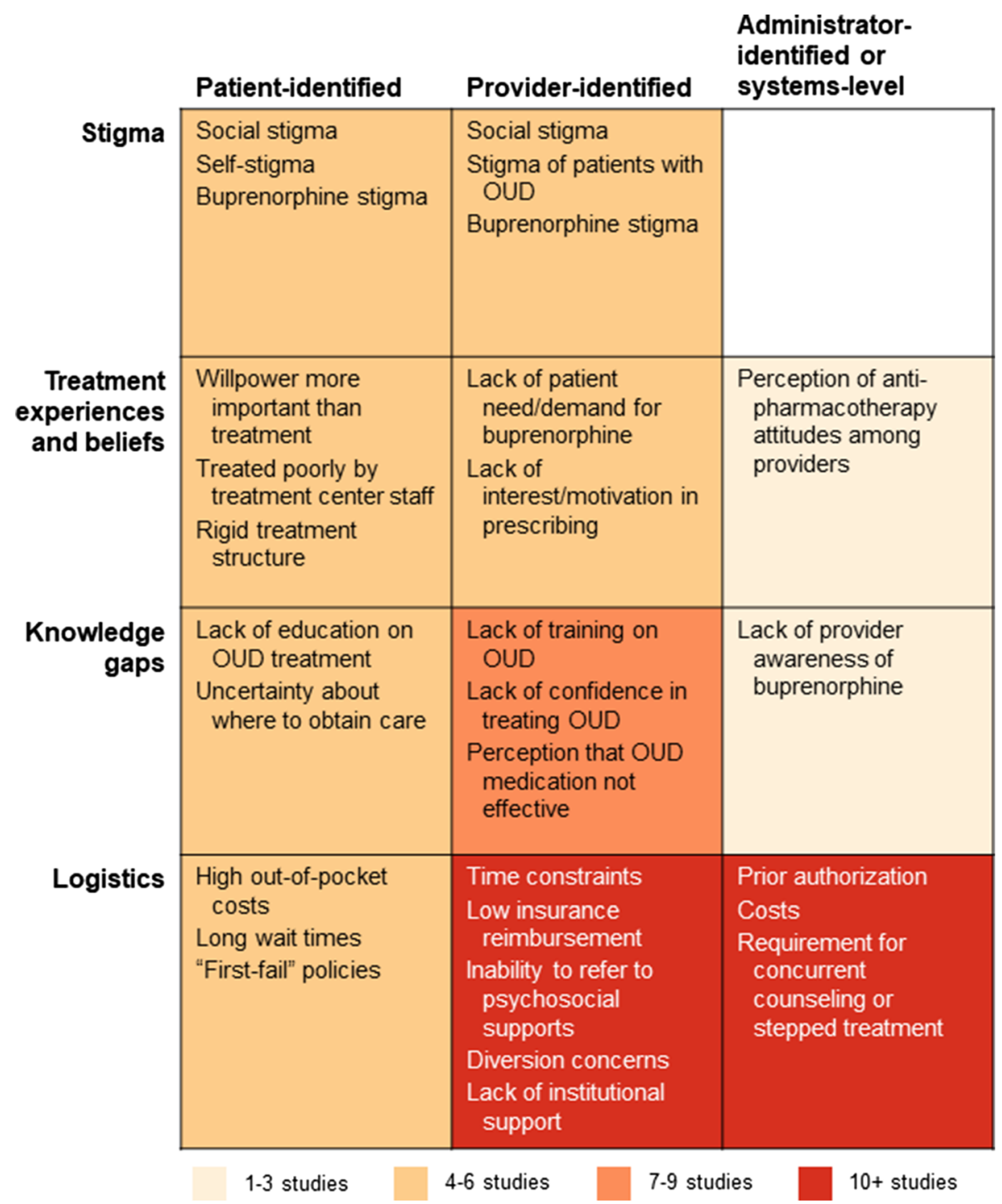

Figure 2 Heat map of barriers to buprenorphine and naltrexone identified by patients, providers, and administrators/systems. This heat map illustrates the categories of barriers (stigma, treatment experiences/beliefs, knowledge gaps, and logistics) most frequently cited and by whom (patient-identified, provider-identified, and administrator-identified/system-level) in included studies. The text within each cell represents examples of specific barriers that fell within each category (i.e., these do not represent a comprehensive list of all barriers discussed within these categories).

with a history of OUD treatment (one of the patients at a syringe exchange program in New York City and the other in Alaska) discussed negative experiences, including being treated poorly by treatment center staff, not feeling supported by staff, or not trusting staff. ${ }^{21,23}$ Participants in 2 studies cited prior use of illicit buprenorphine as a negative experience (likely due to concurrent use with opioids which would precipitate withdrawal). ${ }^{21,}{ }^{39}$ In a qualitative study based on interviews with previously incarcerated individuals at addiction treatment centers, some participants stated that a reason why they had not sought OUD treatment in the past was a belief that personal characteristics such as willpower and readiness to change are more important to opioid abstinence than medical treatment $\mathrm{t}^{22}$ - a perspective that may reflect overlapping barriers of stigma and treatment experiences.
Patients also noted several logistical barriers to starting OUD medication. High out-of-pocket costs, including insurance copays and costs associated with "cash-only" providers who do not accept insurance, was a frequently cited barrier. Patients also identified logistical barriers such as difficulty locating a buprenorphine provider, provider waiting lists, delays to treatment initiation, policies that require failing abstinence-based treatment prior to receiving buprenorphine, and practical issues such as not having access to transportation and childcare to attend treatment visits. Participants in 4 studies identified lack of knowledge and education regarding treatment options as barriers. ${ }^{21-23,39}$

Only one study examined patient-identified barriers to naltrexone. ${ }^{39}$ In this study of patients with OUD taking 
Table 1 Categories and Sub-categories of Patient-Identified Barriers and Facilitators

\begin{tabular}{|c|c|c|}
\hline Category (n) & Barrier sub-categories (n) & Facilitator sub-categories (n) \\
\hline Stigma $(6)^{17,21-23,37,39}$ & $\begin{array}{l}\text { Social stigma (4) } \\
\text { Example-Fear of judgment from others if they knew } \\
\text { patient was an addict, not wanting to be seen at treatment } \\
\text { center } \\
\text { Self or internalized stigma (1) } \\
\text { Example-Patients' feelings about themselves as they } \\
\text { try to access treatment, including the experience of shame } \\
\text { related to having OUD and needing treatment } \\
\text { Stigma specific to buprenorphine use (4) } \\
\text { Example-Buprenorphine as a "crutch" or substituting one } \\
\text { addiction for another; belief that people on MAT were still } \\
\text { addicts and not in the recovery process }\end{array}$ & $\begin{array}{l}\text { Positive social support from peers } \\
\text { and family (3) }\end{array}$ \\
\hline $\begin{array}{l}\text { Treatment experiences and } \\
\text { beliefs }(6)^{17,21-23,39,41}\end{array}$ & $\begin{array}{l}\text { Belief that individual traits like willpower and readiness for } \\
\text { change are more important than treatment }(1) \\
\text { Negative experience with prior treatment or treating } \\
\text { providers (1) } \\
\text { Example-Patients treated poorly by treatment center staff/provider; } \\
\text { lack of trust in physician/ mistrust in patients }\end{array}$ & $\begin{array}{l}\text { Helps prevent re-incarceration (1) } \\
\text { Support from treatment providers (1) } \\
\text { Benefits in } \\
\text { symptom relief, functional } \\
\text { improvement, } \\
\text { and treatment of comorbidities } \\
\text { such as pain (1) } \\
\text { Medications can help prevent relapse } \\
\text { and risk of overdose (1) } \\
\text { Rigid treatment structure (positive } \\
\text { experience) (2) } \\
\text { Use of illicit buprenorphine } \\
\text { (positive experience) (3) }\end{array}$ \\
\hline Knowledge gaps $(4)^{21-23,39}$ & $\begin{array}{l}\text { Unsure where to obtain care (1) } \\
\text { Lack of education on drugs and OUD treatment (1) } \\
\text { Unsure what to expect with naltrexone and long-acting } \\
\text { forms of buprenorphine }(1) \\
\text { Misunderstanding how co-formulated naloxone affects } \\
\text { treatment (1) }\end{array}$ & None \\
\hline Logistics $(5)^{17,23,37,21,39}$ & $\begin{array}{l}\text { Out-of-pocket costs, including "cash-only" providers (4) } \\
\text { Finding a provider who accepts insurance and/or } \\
\text { accepting new patients, long wait time (3) } \\
\text { Need to "first fail" abstinence-based treatment (1) } \\
\text { Transportation and childcare barriers (1) } \\
\text { Need to stop other opioids before starting buprenorphine, } \\
\text { delays in treatment benefits (1) } \\
\text { Requirement for urine drug testing prior to treatment } \\
\text { initiation (1) }\end{array}$ & $\begin{array}{l}\text { More time and money once not } \\
\text { obtaining illicit opioids (1) }\end{array}$ \\
\hline
\end{tabular}

buprenorphine or methadone, participants identified not being ready to stop all opioids as a primary barrier to starting naltrexone with related concerns about its effectiveness and ability to manage withdrawal symptoms and pain.

\section{Patient-Identified Facilitators to the Use of OUD Medications}

The most commonly cited facilitator of initiating buprenorphine treatment was receiving personal support from peers, family, and treatment providers. Peers can also be a source of knowledge regarding where and how to access OUD medications. ${ }^{41}$ Participants in 3 studies also reported positive experiences with illicit buprenorphine to manage cravings and withdrawal as a facilitator to seeking treatment with prescribed buprenorphine. ${ }^{17,21,22}$ Participants in one study discussed the potential of both buprenorphine and naltrexone to prevent the risk of relapse and the use of buprenorphine specifically to prevent overdose as reasons for initiating treatment. $^{41}$

\section{Provider-Identified Barriers to Prescribing OUD Medications}

Table 2 provides an overview of provider-identified barriers and facilitators. Logistical issues were the most common types of barriers identified by providers. Lack of time was identified as a barrier in $9 / 14$ studies of providers and was the first or second most frequently cited barrier in 5 studies. ${ }^{19,} 24,26,28,29$ In an online survey of US physicians, $29 \%$ of physicians who did not have a buprenorphine waiver cited concerns about being "inundated" with requests for buprenorphine as a reason for not prescribing it. ${ }^{24}$ Similarly, among a group of waivered providers $(N=272)$ who were not prescribing at maximal capacity (i.e., prescribing up to patient caps of 30 and 100), insufficient time was the most commonly cited reason for not taking on more patients. ${ }^{24}$ Other logistical barriers identified by providers included concerns about insurance reimbursement, need for prior authorizations, and regulatory factors such as Drug Enforcement Agency (DEA) oversight.

Providers in 6 studies cited stigma as a barrier to prescribing OUD medications ${ }^{11-13,19,24,45}$ Stigma among providers can 
Table 2 Categories and Sub-categories of Provider-Identified Barriers and Facilitators

\begin{tabular}{|c|c|c|}
\hline Category (n) & Barriers subcategory (n) & Facilitators subcategory (n) \\
\hline Stigma $(6)^{11-13,19,24,45}$ & $\begin{array}{l}\text { Social stigma (5) } \\
\text { Example-Fear of becoming community } \\
\text { addiction/buprenorphine provider, "not wanting } \\
\text { to attract drug users to your practice" } \\
\text { Perception of patients with OUD (2) } \\
\text { Example-Patients with addiction are "difficult"; } \\
\text { mistrust of patients } \\
\text { Stigma specific to buprenorphine use (4) } \\
\text { Example-Buprenorphine is substituting one } \\
\text { addiction for another }\end{array}$ & None \\
\hline $\begin{array}{l}\text { Treatment experiences and beliefs } \\
(4)^{11,19,24,27}\end{array}$ & $\begin{array}{l}\text { Perception of lack of patient need or demand } \\
\text { for buprenorphine (2) } \\
\text { Lack of interest in prescribing (1) } \\
\text { Lack of motivation to start patients on buprenorphine } \\
\text { in ED setting as providers don't see patients again (1) }\end{array}$ & $\begin{array}{l}\text { Recognizing patient need/demand for } \\
\text { buprenorphine }(2) \\
\text { Create tracking system so ED providers } \\
\text { can track patients over time (1) }\end{array}$ \\
\hline $\begin{array}{l}\text { Knowledge }(9)^{11,12,14,15,19,24,} \\
\text { Ka, } 35\end{array}$ & $\begin{array}{l}\text { Lack of training on OUD or OUD medications or lack } \\
\text { of confidence in ability to treat OUD ( } 7) \\
\text { Perception that OUD medications are not effective (2) } \\
\text { Perception that patients do not need OUD medications } \\
(2) \\
\text { Not knowing how to obtain waiver (1) }\end{array}$ & $\begin{array}{l}\text { Mentoring (2) } \\
\text { Access to education and training (2) }\end{array}$ \\
\hline 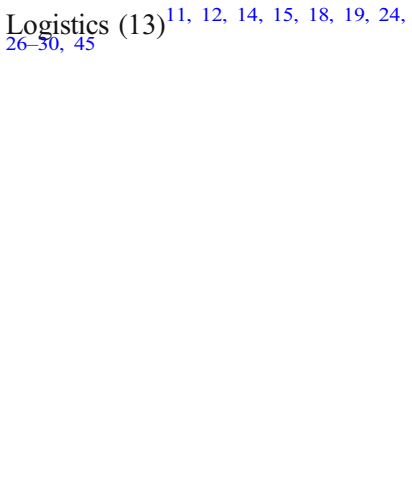 & $\begin{array}{l}\text { Low insurance reimbursement or need for prior } \\
\text { authorizations (9) } \\
\text { Inability to refer to psychosocial supports, } \\
\text { lack of referral/collaboration with addiction specialist, } \\
\text { or inability to follow-up with patients after ED } \\
\text { buprenorphine induction ( } 8 \text { ) } \\
\text { Concerns about diversion ( } 7) \\
\text { Lack of practice partner and/or institutional support (5) } \\
\text { Lack of staff resources or space or fear of increased } \\
\text { volume of patients (4) } \\
\text { Cumbersome regulatory requirements (4) } \\
\text { Geographic distance (1) }\end{array}$ & $\begin{array}{l}\text { Information about/ability to refer to specialty } \\
\text { care (2) or use of EHR and individualized } \\
\text { care plans for follow-up after ED induction (1) } \\
\text { Presence of peer and institutional support (2) } \\
\text { Have clear clinical protocols for buprenorphine } \\
\text { induction in ED settings (1) } \\
\text { Telehealth-delivered OUD treatment (1) }\end{array}$ \\
\hline
\end{tabular}

manifest as not wanting to be known as an OUD treatment provider, ${ }^{11}$ concerns about patients with OUD negatively impacting staff or other patients, ${ }^{45}$ and concerns about attracting more patients with drug use to their practice. ${ }^{12}$ Although not explicitly stated by study participants, stigma related to patients with OUD (a perception that patients with OUD are "difficult" or not trust-worthy ${ }^{13,19}$ ) may contribute to providers' practical concerns about the time needed to provide OUD treatment as well as concerns about medication misuse and diversion, which was cited as a barrier in 8 studies. ${ }^{11,12,14,19,24,28-30}$

Study participants also identified OUD knowledge gaps as barriers to prescribing. In a qualitative study of 20 physicians, $75 \%$ cited lack of addiction education in medical school and residency as a barrier to prescribing OUD medications. ${ }^{11}$ Lack of knowledge can also translate into low confidence in prescribing buprenorphine, which was identified as a barrier in 3 studies. $^{12,14,30}$

Lastly, beliefs about OUD treatment, such as the perception that buprenorphine is not effective, that patients have a low need for it, or that it is "substituting one addiction for another," can impact providers' decisions not to prescribe OUD medication. ${ }^{11,19,}{ }^{30}$ A lack of interest or motivation to prescribe buprenorphine was cited as a barrier in 2 studies. ${ }^{19,27}$ As noted in a study that included a mix of healthcare providers and law enforcement agents and judicial officers in Ohio, the legacy of "pill mills" as a contributor to the OUD crisis may also influence attitudes towards using medication to treat OUD. ${ }^{14}$

In the 1 study that examined provider-identified barriers specific to naltrexone, physicians identified knowledge gaps in using naltrexone for OUD treatment and concerns about patient willingness to stop other opioids in order to start naltrexone without access to medically-supervised detoxification programs. ${ }^{11}$ Gaps in knowledge of OUD medication were also identified in a qualitative study on use and acceptability of OUD medications among 725 addiction counselors in substance abuse treatment centers, which found that about $20 \%$ of counselors could not rate the effectiveness of buprenorphine and that $90 \%$ of this group $(N=138)$ had received little to no medication-specific training. ${ }^{10}$ Another qualitative study of 47 addiction professionals (83\% counselors) found that those who subscribed to abstinence-based approaches to OUD treatment disagreed with the use of OUD medications. ${ }^{32}$ Although indirectly related to primary care and other non-specialized outpatient settings, the perspectives of addiction counselors may influence patients' treatment decisions. Similarly, 
Table 3. Categories and Sub-categories of System-Level Barriers and Facilitators

\begin{tabular}{|c|c|c|}
\hline$\overline{\text { Category (n) }}$ & Barriers subcategory $(n)$ & Facilitators subcategory $(n)$ \\
\hline \multicolumn{3}{|l|}{ Stigma (0) } \\
\hline Treatment experiences and beliefs $(2)^{35,36}$ & $\begin{array}{l}\text { Anti-pharmacotherapy attitudes } \\
\text { among providers }(2)\end{array}$ & $\begin{array}{l}\text { Anti-pharmacotherapy attitude } \\
\text { diminished over time as providers } \\
\text { gained experience with medications }\end{array}$ \\
\hline Knowledge $(1)^{36}$ & $\begin{array}{l}\text { Lack of provider awareness/understanding } \\
\text { of buprenorphine (1) }\end{array}$ & Provider knowledge of buprenorphine (1) \\
\hline Logistics (10) $16,20,25,33,35,36,40,42,44,48$ & $\begin{array}{l}\text { Requirement of prior authorization (3) } \\
\text { Costs (including copayments) (3) } \\
\text { Requirement for concurrent counseling } \\
\text { or stepped treatment (2) } \\
\text { Provider concerns about diversion (2) } \\
\text { Certain medications and formulations not covered } \\
\text { (e.g., naltrexone not covered, injectable or } \\
\text { implantable formulations not covered) (1) } \\
\text { Limited provider availability/capacity (1) } \\
\text { Providers do not accept insurance for buprenorphine (1) } \\
\text { Patients lack health insurance (1) }\end{array}$ & $\begin{array}{l}\text { Coverage of all medications/ } \\
\text { treatment for OUD (2) } \\
\text { Medicaid expansion (2) }\end{array}$ \\
\hline
\end{tabular}

perspectives of correctional facility staff may also influence OUD treatment decisions given the high prevalence of criminal justice system involvement among patients with OUD. ${ }^{22}$ In a survey of correctional facility employees $(N=959)$, decisions to refer patients to OUD medication treatment were associated level of education and training, work setting (treatment or corrections-based), and the extent to which employees viewed medication as "trading one addiction for another."

\section{Provider-Identified Facilitators to OUD Medication Prescribing}

Five studies described provider-identified facilitators to prescribing buprenorphine, which included patient demand, streamlined clinical protocols and referral pathways, increased reimbursement, training in OUD treatment, peer supports, and mentorship. Among these, study participants most frequently cited a recognition of patient need and/or demand for OUD medications, peer and institutional supports, and mentoring as facilitators.

\section{Administrator-Identified and System-Level Bar- riers and Facilitators}

Eleven studies ${ }^{16,20,25,33-36,40,42,44,48}$ examined barriers and facilitators to OUD medication utilization at the system level, either through interviews or surveys of healthcare administrators or through analysis of claims data or insurance plans (Table 3). Barriers identified in these studies predominantly fell into the category of logistical or practical issues and echoed barriers identified by patients and providers. One of the most frequently cited of these barriers was the need for prior authorization before OUD medications can be prescribed. ${ }^{16,25,33,42}$ Tellingly, one national survey of commercial health plans found that as the percent of plans who cover OUD medications has increased (65 to $97 \%$ ), so has the percent of plans that required prior authorization (8 to $39 \%) .{ }^{42}$ Other barriers have persisted in limiting patients' access to OUD medications even when insured. For example, one survey of 327 active buprenorphine prescribers in Ohio reported that only half accepted insurance for buprenorphine treatment. ${ }^{40}$ Another study found that of 100 marketplace health plans, not all covered all types of OUD medications, with naltrexone less frequently covered than buprenorphine and injectable drugs less frequently covered than oral drugs. ${ }^{25}$

Expansion of insurance coverage was the most frequently cited facilitator to OUD medication use among system-level studies. On a national level, the rates of payers that cover OUD medications has steadily increased over time $(65 \%$ of commercial plans covered buprenorphine in 2003 vs $97 \%$ in $2014 ;{ }^{42} 29 / 50$ state Medicaid programs covered buprenorphine in 2004 vs. $40 / 50$ in $2013^{16}$ ). Unsurprisingly, when more patients have access to insurance, more patients receive OUD medications. A survey of state Medicaid offices found that the 26 states that participated in the ACA Medicaid expansion saw a $70 \%$ increase in Medicaid-covered buprenorphine prescriptions and a 50\% increase in buprenorphine spending ${ }^{48}$ compared to those that did not expand Medicaid. Along the same lines, a database study of 1,748 injection drug users in Maryland found that those who had access to health insurance had 3 times the odds of receiving a buprenorphine prescription than those who did not. ${ }^{20}$

\section{DISCUSSION}

Expanding access to medications for OUD is a critically important component of the public health effort to reduce opioid-related morbidity, mortality, and misuse. In this systematic review of the most recent evidence on barriers and facilitators associated with OUD medication use, we found that patient-, provider-, and system-level barriers fell into 4 categories - stigma, treatment experiences and beliefs, practical or logistical issues, and knowledge of the role of medications in OUD treatment. Overall, we found that stigma and negative treatment experiences were the most common 
barriers identified by patients, while practical and logistical issues related to time, insurance requirements, and regulatory factors including DEA oversight were the most common barriers identified by providers. Administrator-identified or system-level barriers echoed the logistical barriers identified by both patients and providers.

The provider-level barriers to OUD medication use identified in this review are largely the same as those identified in a qualitative study of administrative and clinical staff at VHA sites in 2006-2007, which found the main barriers to be lack of education regarding buprenorphine treatment, negative perceptions of patients with OUD, perceived lack of resources, and the thought that OUD care was best delivered outside the VA (presumably in specialized settings). ${ }^{49}$ This overlap in findings from 2006 to 2007 and from evidence 2014forward suggests that despite federal policy advancements, certain barriers including stigma have an enduring role in the underutilization of OUD medications.

Although the studies in this review were not designed to evaluate causation or associations among specific barriers, it is plausible that the barriers encountered by patients and providers, which are reinforced by system-level barriers, have an additive effect resulting in fewer patients with OUD starting medication. In particular, we suspect that stigma related to OUD, identified as a barrier by patients and providers, may underlie other barrier types. For example, a perception that patients with OUD are difficult to treat may add to provider concerns about the time needed to prescribe OUD medications, particularly buprenorphine, as well as concerns about diversion and liability. The historical precedent of highly regulating substance abuse treatment and stigma at the institutional level may contribute to several system-level barriers including insurance requirements and reimbursement rates, as well as to perceptions of OUD treatment as cumbersome and difficult to implement in non-specialized settings.

Facilitators of OUD medication use for both patients and providers include peer support and knowledge-sharing. These findings highlight the potential for community - peer supports for patients and communities of practice for providers - to overcome some of the barriers to OUD medication use including stigma and knowledge gaps. For example, provider education related to the reasons why most patients use diverted buprenorphine (to manage withdrawal and cravings) and mentoring by addiction treatment specialists on harm reduction approaches may reduce concerns related to buprenorphine diversion. Two examples of structured provider support are the VA's SCOUTT (Stepped Care for Opioid Use Disorder Train the Trainer) initiative that provides training and support of interdisciplinary teams to deliver a stepped model of OUD care, and the ECHO (Extension for Community Healthcare Outcomes) model that links a "hub" of treatment addiction specialists to "spokes" of community-based providers through telehealth-based consults. ${ }^{15,45,50,51}$

The studies included in this review have several limitations. First, studies predominantly included patients with a history of injection drug use who were engaged in treatment and may not be generalizable to those with OUD related to prescription opioids and/or those with OUD who are not treatment-engaged. Second, although half of studies met our minimum quality criteria, several survey studies had low $(3-46 \%)^{19,24,28-30}$ or unclear $^{21-23}$ response rates. In general, low survey response rates may lead to nonresponse bias, in which those who respond differ in meaningful ways from those who do not respond. It is difficult to tell how low response rates affected our review's conclusions. Third, results do not tell us the size or severity of a barrier or facilitator, its relative impact on treatment decisions, or how barriers and facilitators impact each other. Fourth, no studies directly evaluated whether barriers and facilitators vary by patient or provider characteristics or setting.

In terms of our rapid review methods, limitations include our use of second reviewer checking in lieu of dual independent review which may have resulted in missing relevant studies or data. However, we likely captured the major barrier and facilitator types given that many of the studies discussed the same themes. Second, using a streamlined approach to assessing study quality means that we could not definitively determine which studies were the highest quality. However, given the similarities among study findings, identifying the highest-quality studies would have been unlikely to change our conclusions. Third, limiting our review to studies published in 2014 or later may have missed important studies published in 2013 or earlier. However, we scanned the pre-2014 literature and found that the most relevant study was a qualitative study of VHA administrative and clinical staff in 2006-2007 that identified similar barrier types as our review. ${ }^{49}$ Fourth, because study heterogeneity limited our ability to synthesize evidence on the relative importance of each barrier and facilitator, we used the proportion of studies that reported a certain barrier or facilitator as a proxy for their relevant importance. Other reviewers may take a different approach and come to different conclusions about groupings of barriers and facilitators and/or which are most important.

In conclusion, stigma, treatment experiences and beliefs, logistical issues, and knowledge gaps are associated with low utilization of OUD medications among patients and providers. System-level barriers are largely the same as the logistical and practical barriers identified by patients and providers. These barriers can overlap and mutually reinforce each other, but given that, it is also plausible that reducing one barrier may lead to reductions in others. The highest priority for future research is to evaluate interventions to reduce stigma. Other priorities for future research include better identification of barriers and facilitators for specific populations, such as those with OUD related to prescription opioids, and for naltrexone use.

Acknowledgments: The authors gratefully acknowledge Mark Helfand, MD, MPH, MS, for methodological and clinical input; Karen Drexler, MD; and Ellen Edens, MD, MPH, MA, for input on scope; Payten Sonnen, BA, for editorial support; and Julia Haskin MA for graphic design of Figure 2. 
Corresponding Author: Katherine Mackey, MD, MPP; Evidence Synthesis Program (ESP) Coordinating Center, VA Portland Health Care System, Portland, OR, USA (e-mail: katherine.mackey@va.gov).

Funding This material is based upon work supported by the Department of Veterans Affairs, Veterans Health Administration, Health Services Research and Development, Evidence Synthesis Program (ESP).

\section{Compliance with Ethical Standards:}

Conflict of Interest: The authors declare that they do not have a conflict of interest.

Disclaimer: The views expressed in this article are those of the authors and do not necessarily represent the views of the Department of Veterans Affairs or the US government.

\section{REFERENCES}

1. Manhapra A, Stefanovics E, Rosenheck R. Initiating opioid agonist treatment for opioid use disorder nationally in the Veterans Health Administration: Who gets what? Subst Abus. 2019:1-11.

2. National Academies of Sciences, Engineering, and Medicine; Health and Medicine Division; Board on Health Sciences Policy; Committee on Medication-Assisted Treatment for Opioid Use Disorder; Mancher M, Leshner AI, eds. Medications for Opioid Use Disorder Save Lives. Washington (DC): National Academies Press (US); 2019. https://doi org/10.17226/25310. Available from: https://www.ncbi.nlm.nih.gov/ books/NBK538936/. Accessed Jun 19, 2020.

3. President Donald J. Trump is Combatting the Opioid Crisis. The Whitehouse https://www.whitehouse.gov/briefings-statements/president-donald-j-trump-combatting-opioid-crisis/. Published 2018. Accessed Jun 19, 2020.

4. Mackey K, Veazie S, Anderson J, Bourne D, Peterson K. Evidence Brief: Barriers and Facilitators to Use of Medications for Opioid Use Disorder. Evidence Synthesis Program, Health Services Research and Development Service, Office of Research and Development, Department of Veterans Affairs; 2019. Available from: https://www.hsrd.research.va. gov/publications/esp/reports.cfm. Accessed 19 Jun 2020

5. Berkman ND, Lohr KN, Ansari M, et al. Grading the Strength of a Body of Evidence When Assessing Health Care Interventions for the Effective Health Care Program of the Agency for Healthcare Research and Quality: An Update Methods Guide for Effectiveness and Comparative Effectiveness Reviews. Rockville, MD; 2013.

6. Moher D, Liberati A, Tetzlaff J, Altman DG. Preferred reporting items for systematic reviews and meta-analyses: the PRISMA statement. BMJ. 2009;339:b2535.

7. Methods Guide for Effectiveness and Comparative Effectiveness Reviews. Rockville, MD: Agency for Healthcare Research and Quality; 2014.

8. Hannes $\mathbf{K}$. Supplementary guidance for inclusion of qualitative research in Cochrane systematic reviews of interventions; 2011.

9. The Joanna Briggs Institute. Critical Appraisal Tools; 2017. https:// joannabriggs.org/critical_appraisal_tools. Accessed July, 2019

10. Aletraris L, Edmond MB, Paino M, Fields D, Roman PM. Counselor training and attitudes toward pharmacotherapies for opioid use disorder. Subst Abus. 2016;37(1):47-53

11. Andraka-Christou B, Capone MJ. A qualitative study comparing physician-reported barriers to treating addiction using buprenorphine and extended-release naltrexone in U.S. office-based practices. Int J Drug Policy. 2018;54:9-17.

12. Andrilla CHA, Coulthard C, Larson EH. Barriers Rural Physicians Face Prescribing Buprenorphine for Opioid Use Disorder. Ann Fam Med. 2017;15(4):359-362.

13. Andrilla CHA, Coulthard C, Patterson DG. Prescribing Practices of Rural Physicians Waivered to Prescribe Buprenorphine. Am J Prev Med. 2018;54(6):S208-S214.

14. Andrilla CHA, Jones KC, Patterson DG. Prescribing Practices of Nurse Practitioners and Physician Assistants Waivered to Prescribe Buprenorphine and the Barriers They Experience Prescribing Buprenorphine. J Rural Health. 2020;36(2):187-195.
15. Brunet N, Moore DT, Lendvai Wischik D, Mattocks KM, Rosen MI. Increasing buprenorphine access for veterans with opioid use disorder in rural clinics using telemedicine. Subst Abus. 2020:1-8.

16. Burns RM, Pacula RL, Bauhoff S, et al. Policies related to opioid agonist therapy for opioid use disorders: The evolution of state policies from 2004 to 2013. Subst Abus. 2016;37(1):63-69.

17. Cicero TJ, Ellis MS, Chilcoat HD. Understanding the use of diverted buprenorphine. Drug Alcohol Depend. 2018;193:117-123.

18. Cooper HL, Cloud DH, Freeman PR, et al. Buprenorphine dispensing in an epicenter of the U.S. opioid epidemic: A case study of the rural risk environment in Appalachian Kentucky. Int J Drug Policy. 2020.

19. DeFlavio JR, Rolin SA, Nordstrom BR, Kazal LA, Jr. Analysis of barriers to adoption of buprenorphine maintenance therapy by family physicians. Rural Remote Health. 2015;15:3019.

20. Feder KA, Krawczyk N, Mojtabai R, Crum RM, Kirk G, Mehta SH. Health insurance coverage is associated with access to substance use treatment among individuals with injection drug use: Evidence from a 12-year prospective study. J Subst Abus Treat. 2019;96:75-81.

21. Fox AD, Chamberlain A, Sohler NL, Frost T, Cunningham Co. Illicit buprenorphine use, interest in and access to buprenorphine treatment among syringe exchange participants. J Subst Abus Treat. 2015;48(1):112-116.

22. Fox AD, Maradiaga J, Weiss L, Sanchez J, Starrels JL, Cunningham Co. Release from incarceration, relapse to opioid use and the potential for buprenorphine maintenance treatment: a qualitative study of the perceptions of former inmates with opioid use disorder. Addict Sci Clin Pract. 2015;10:2.

23. Hewell VM, Vasquez AR, Rivkin ID. Systemic and individual factors in the buprenorphine treatment-seeking process: a qualitative study. Subst Abuse Treat Prev Policy. 2017;12(1):3.

24. Huhn AS, Dunn KE. Why aren't physicians prescribing more buprenorphine? J Subst Abus Treat. 2017;78:1-7.

25. Huskamp HA, Riedel LE, Barry CL, Busch AB. Coverage of Medications That Treat Opioid Use Disorder and Opioids for Pain Management in Marketplace Plans, 2017. Med Care. 2018;56(6):505-509.

26. Hutchinson E, Catlin M, Andrilla C, Baldwin L-M, Rosenblatt RA. Barriers to primary care physicians prescribing buprenorphine. Ann Fam Med. 2014;12(2):128-133.

27. Im DD, Chary A, Condella AL, et al. Emergency Department Clinicians' Attitudes Toward Opioid Use Disorder and Emergency Departmentinitiated Buprenorphine Treatment: A mixed-methods study. West J Emerg Med. 2020;21(2):261-271.

28. Jones CM, McCance-Katz EF. Characteristics and prescribing practices of clinicians recently waivered to prescribe buprenorphine for the treatment of opioid use disorder. Addiction. 2019;114(3):471-482.

29. Kermack A, Flannery M, Tofighi B, McNeely J, Lee JD. Buprenorphine prescribing practice trends and attitudes among New York providers. J Subst Abus Treat. 2017;74:1-6.

30. Kirane H, Drits E, Ahn S, et al. Addressing the opioid crisis: An assessment of clinicians' training experience, practices, and attitudes within a large healthcare organization. J Opioid Manag. 2019;15(3):193-204.

31. Lagisetty PA, Bohnert A. Role of an accurate treatment locator and cash-only practices in access to buprenorphine for opioid use disorders. Ann Intern Med. 2019;171(1):58-59.

32. Madden EF. Intervention stigma: How medication-assisted treatment marginalizes patients and providers. Soc Sci Med. 2019;232:324-331

33. Mark TL, Parish WJ, Zarkin GA. Association of Formulary Prior Authorization Policies With Buprenorphine-Naloxone Prescriptions and Hospital and Emergency Department Use Among Medicare Beneficiaries. JAMA Netw Open. 2020;3(4):e203132.

34. McClellan C, Fingar KR, Ali MM, Olesiuk WJ, Mutter R, Gibson TB. Price elasticity of demand for buprenorphine/naloxone prescriptions. J Subst Abus Treat. 2019;106:4-11.

35. Molfenter T, Fitzgerald M, Jacobson N, McCarty D, Quanbeck A, Zehner M. Barriers to Buprenorphine Expansion in Ohio: A TimeElapsed Qualitative Study. J Psychoactive Drugs. 2019:1-8.

36. Molfenter T, Sherbeck C, Zehner $\mathbf{M}$, et al. Implementing buprenorphine in addiction treatment: payer and provider perspectives in Ohio. Subst Abuse Treat Prev Policy. 2015;10:13.

37. Monico LB. A systems perspective of buprenorphine patients' experiences in an opioid treatment program. Dissertation Abstracts International Section A: Humanities and Social Sciences. 2017;78(1-A(E)):No Pagination Specified.

38. Murphy SM, Fishman PA, McPherson S, Dyck DG, Roll JR. Determinants of buprenorphine treatment for opioid dependence. J Subst Abus Treat. 2014;46(3):315-319. 
39. Muthulingam D, Bia J, Madden LM, Farnum So, Barry DT, Altice FL. Using nominal group technique to identify barriers, facilitators, and preferences among patients seeking treatment for opioid use disorder: A needs assessment for decision making support. J Subst Abus Treat. 2019;100:18-28.

40. Parran TV, Muller JZ, Chernyak E, et al. Access to and Payment for Office-Based Buprenorphine Treatment in OH. Subst Abuse. 2017;11(no pagination).

41. Randall-Kosich O, Andraka-Christou B, Totaram R, Alamo J, Nadig M. Comparing Reasons for Starting and Stopping Methadone, Buprenorphine, and Naltrexone Treatment Among a Sample of White Individuals With Opioid Use Disorder. J Addict Med. 2019.

42. Reif S, Acevedo A, Garnick DW, Fullerton CA. Reducing Behavioral Health Inpatient Readmissions for People With Substance Use Disorders: Do Follow-Up Services Matter? Psychiatr Serv. 2017;68(8):810-818.

43. Richard EL, Schalkoff CA, Piscalko HM, et al. "You are not clean until you're not on anything": Perceptions of medication-assisted treatment in rural Appalachia. Int J Drug Policy. 2020.

44. Saloner B, Levin J, Chang H-Y, Jones C, Alexander GC. Changes in Buprenorphine-Naloxone and Opioid Pain Reliever Prescriptions After the Affordable Care Act Medicaid Expansion. JAMA Netw Open. 2018;1(4):e181588-e181588.

45. Shea CM, Gertner AK, Green SL. Barriers and perceived usefulness of an ECHO intervention for office-based buprenorphine treatment for opioid use disorder in North Carolina: A qualitative study. Subst Abus. 2019:1-11.

46. Simon CB, Tsui JI, Merrill JO, Adwell A, Tamru E, Klein JW. Linking patients with buprenorphine treatment in primary care: Predictors of engagement. Drug Alcohol Depend. 2017;181:58-62.

47. Streisel S. Intent to refer: Exploring bias toward specific medicationassisted treatments by community corrections employees. Subst Use Misuse. 2018;53(14):2421-2430.

48. Wen H, Hockenberry JM, Borders TF, Druss BG. Impact of Medicaid Expansion on Medicaid-covered Utilization of Buprenorphine for Opioid Use Disorder Treatment. Med Care. 2017;55(4):336-341.

49. Gordon AJ, Kavanagh G, Krumm M, et al. Facilitators and barriers in implementing buprenorphine in the Veterans Health Administration. Psychol Addict Behav. 2011;25(2):215-224.

50. Department of Veterans Affairs, Quality Enhancement Research Initiative. Facilitation of the Stepped-Care Model and Medication Treatment for Opioid Use Disorder. https://www.queri.research.va.gov/national partnered_evaluations/oud.cfm. Accessed July 15, 2019.

51. Hagedorn H, Kenny M, Gordon AJ, et al. Advancing pharmacological treatments for opioid use disorder (ADaPT-OUD): protocol for testing a novel strategy to improve implementation of medication-assisted treatment for veterans with opioid use disorders in low-performing facilities. Addict Sci Clin Pract. 2018;13(1):25.

Publisher's Note: Springer Nature remains neutral with regard to jurisdictional claims in published maps and institutional affiliations. 